Article

\title{
A Marine Fibrinolytic Compound FGFC1 Stimulating Enzymatic Kinetic Parameters of a Reciprocal Activation System Based on a Single Chain Urokinase-Type Plasminogen Activator and Plasminogen
}

\author{
Ruihua Guo 1,2,t, Dong Duan 1,t, Shaotong Hong ${ }^{1}$, Yu Zhou ${ }^{1}$, Fang Wang ${ }^{1}$, Shujun Wang ${ }^{3}$, \\ Weihui $\mathrm{Wu}^{1,3, *}$ and Bin Bao ${ }^{1,2, *}$ \\ 1 College of Food Science and Technology, Shanghai Ocean University, Shanghai 201306, China; \\ rhguo@shou.edu.cn (R.G.); yzhou@shou.edu.cn (Y.Z.); fwang@shou.edu.cn (F.W.) \\ 2 Shanghai Engineering Research Center of Aquatic-Product Processing \& Preservation, \\ Shanghai 201306, China \\ 3 Jiangsu Key Laboratory of Marine Pharmaceutical Compound Screening, Huaihai Institute of Technology, \\ Lianyungang 222005, China; wangsj@hit.edu.cn \\ * Correspondence: whwu@shou.edu.cn (W.W.); bbao@shou.edu.cn (B.B.); \\ Tel.: +86-021-61900388 (W.W.); Tel.: +86-021-61900380 (B.B.) \\ + Both authors contributed equally to this work.
}

\begin{abstract}
A marine fibrinolytic compound FGFC1 enhancing fibrinolysis was obtained involving enzymatic kinetic parameters of a reciprocal activation system with a single chain urokinase type plasminogen activator and plasminogen. FGFC1, a kind of bisindole alkaloid from a metabolite of the rare marine fungi Starchbotrys longispora FG216, modulated enzymatic kinetic parameters including the fibrinolytic reaction rate and fibrin degradation characteristics. The enzymatic kinetics of fibrinolysis was described based on the enzymatic reaction of a chromogenic-substrate associated with $p$-nitroaniline ( $p$-NA). While the single chain urokinase-type plasminogen activator (pro-uPA) activated plasminogen, $k_{\text {cat }}$ and $k_{\text {cat }} / K_{\mathrm{m}}$ increased significantly with an increase of FGFC1 concentration. Moreover, $k_{\mathrm{cat}}$ and $k_{\mathrm{cat}} / K_{\mathrm{m}}$ exhibited 26.5 -fold and 22.8-fold enhanced activity at the concentration of $40 \mu \mathrm{g} \cdot \mathrm{mL}^{-1}$ of FGFC1, respectively. The results suggested that FGFC1 significantly improved the maximum catalytic efficiency and the total catalytic activity of fibrinolysis base on the reciprocal activation of pro-uPA and plasminogen. $K_{m}$ increased with increasing FGFC1 concentration, which indicated that FGFC1 slightly decreased the affinity activity of the pro-uPA and plasminogen versus the enzyme substrate. The marine bisindole alkaloid FGFC1 enhanced fibrinolysis, which was taken on enzymatic kinetic characteristics.
\end{abstract}

Keywords: fibrinolytic activity; FGFC1; plasminogen activator; plasminogen; enzymatic kinetic

\section{Introduction}

The activation between the single chain urokinase-type plasminogen activator (pro-uPA) and plasminogen (plg) plays an important role during the process of fibrinolysis such as start-up, acceleration, and localization [1,2]. As shown in Figure 1, pro-uPA with faint intrinsic activity cleaved the Arg ${ }^{561-V a l}{ }^{562}$ peptide bond of plasminogen [3,4], which was converted to bioactive plasmin (plm). After that, plasmin cleaved the Arg ${ }^{275}-\mathrm{Ile}^{276}$ peptide bond of pro-uPA [5] and converted to more bioactive urokinase (uPA) [6]. Thus, substances that can accelerate its activation has promising applications for the treatment of thrombosis or arteriosclerosis [7]. 


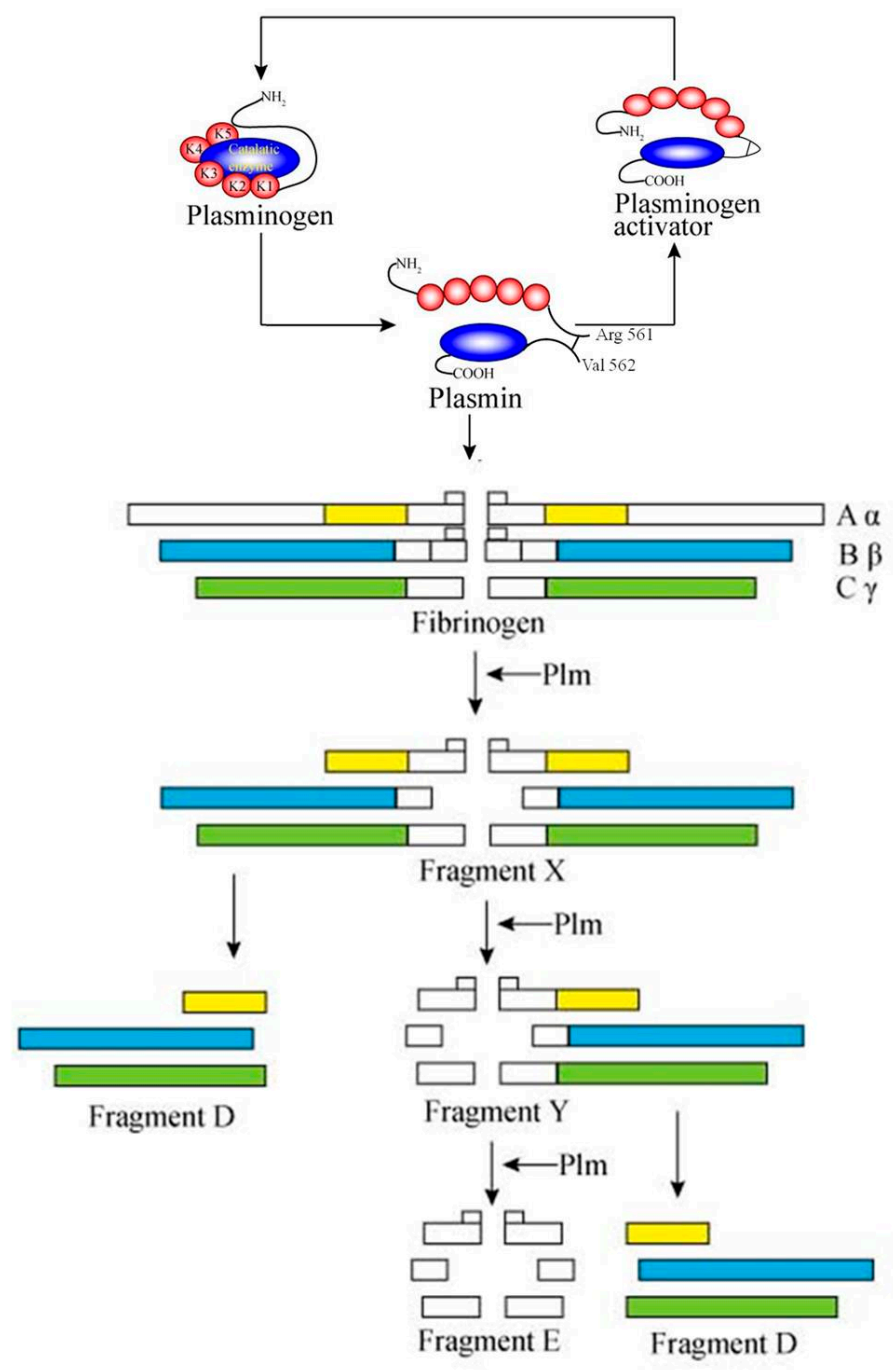

Figure 1. Principle diagram of activation reaction between the pro-uPA and plasminogen in the fibrinolytic process.

Natural products with various structures offer more opportunities to discover lead compounds or drugs $[8,9]$. An isoindolone derivative, Fungi fibrinolytic compound (R)-2,5-bis((2R,3R)-2-((E)-4,8-dimethylnona-3,7-dien-1-yl)-3,5-dihydroxy-2-methyl-7-oxo-3,4,7,9-tetr ahydropyrano[2,3-e]isoindol-8(2H)-yl)pentanoic acid (FGFC1, Fungi fibrinolytic compound 1, Figure 2), was isolated from a rare marine microorganism strain Stachybotrys longispora FG216. This compound was also evaluated for fibrinolytic activity in vitro and in vivo [10,11]. The results showed that FGFC1 could stimulate the generation of plasmin activity by measuring Glu-plasminogen and Lys-plasminogen activation in vitro. The experiment of fluorescein isothiocyanate (FITC)-fibrinogen degradation indicated that the effect of FGFC1 on fibrinolytic activity was mediated by plasminogen and pro-PA. In addition, FGFC1 $(10 \mathrm{mg} / \mathrm{kg})$ could dissolve most of the pulmonary thrombus of Wistar rats in vivo [12]. Previously, we reported that FGFC1 accelerated the mutual activation reaction between a single chain urokinase-type plasminogen activator and plasminogen, and reacted with plasminogen/plasmin, which indicated that FGFC1 accelerated the fibrinolytic process [13]. In the presence of a plasminogen/single chain urokinase type plasminogen activator, the rate of plasminogen converted into plasmin was accelerated. The extent of acceleration can be expressed using plasminogen activation kinetics. The constant kinetics of enzyme-catalyzed reactions can reflect affinity quantitatively and the catalytical activity of the activator to uPA and plasminogen. 
<smiles>CC(C)=CCC/C(C)=C/CC[C@@]1(C)C[C@H](O)c2cc(O)c3c(c2O1)CN(C(CCCN1Cc2c(cc(O)c4c2O[C@](C)(CC/C=C(\C)CCC=C(C)C)C(O)C4)C1=O)C(=O)O)C3=O</smiles>

Figure 2. The chemical structure of FGFC1.

In this paper, the enzymatic reaction kinetics of plasminogen activation by fibrinolytic FGFC1 was described via chromogenic-substrate to research further the effect of FGFC1 on the structure of fibrinolytic factors. $k_{\text {cat }}$ and $k_{\text {cat }} / K_{\mathrm{m}}$ increased significantly with increasing FGFC1 concentration. Moreover, $k_{\text {cat }}$ and $k_{\text {cat }} / K_{\mathrm{m}}$ exhibited 26.5 -fold and 22.8 -fold increased activity at $40 \mu \mathrm{g} \cdot \mathrm{mL}^{-1} \mathrm{FGFC1}$ concentration, respectively. FGFC1 improved the maximum catalytic efficiency and the total catalytic activity of pro-uPA. $K_{\mathrm{m}}$ increased with increasing FGFC1 concentration, which indicated that FGFC1decreased slightly on the affinity activity of pro-uPA versus plasminogen.

\section{Results and Discussion}

\subsection{Kinetic Characteristics of Plasmin}

According to the experimental method, $A_{405} \mathrm{~nm}$ was measured by S-2444 hydrolysis with different concentrations of plasmin $\left(5,10,20,30,40\right.$, and $\left.50 \mathrm{nmol} \cdot \mathrm{L}^{-1}\right)$. The plot of $A_{405}$ versus $t$ of the S-2444 hydrolysis by different concentrations of plasmin is shown in Figure 3. From the equation $K_{n}=$ $\varepsilon \cdot L \cdot k_{\text {cat, }, P l m} \cdot[\mathrm{Plm}]_{n}, K_{n}$ (the slope of each curve) was obtained by the linear regression of $A_{405 \mathrm{~nm}-t}$ in Figure 3, the results of which are shown in Table 1 . The linear slope $K_{n}$ varied from $0.7 \times 10^{-3}$ to $16.3 \times$ $10^{-3} / \mathrm{min}$ with concentrations of plasmin $\left(5-50 \mathrm{nmol} \cdot \mathrm{L}^{-1}\right) \cdot a\left(a=\varepsilon \cdot L \cdot k_{\text {cat,plm }}=3.514 \times 10^{-4}\right.$ $\left.A_{405} \cdot \mathrm{min}^{-1} \cdot \mathrm{nmol}^{-1}\right)$ is constant, and was obtained via linear regression of figure $\left(K_{n}-[\mathrm{Plm}]\right)$ (Figure 4$) \cdot a$ was utilized for the followed experiments.

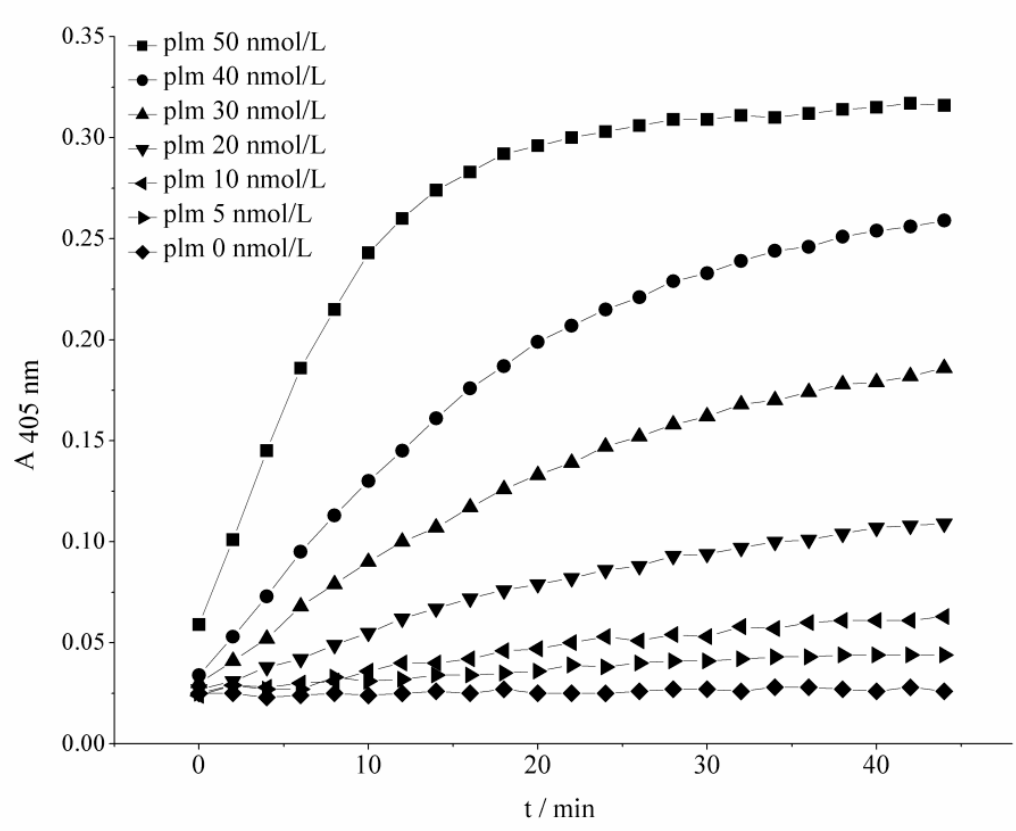

Figure 3. Plot of $A_{405}$ versus $t$ the hydrolysis reaction of S-2444 by different concentrations of plasmin. 


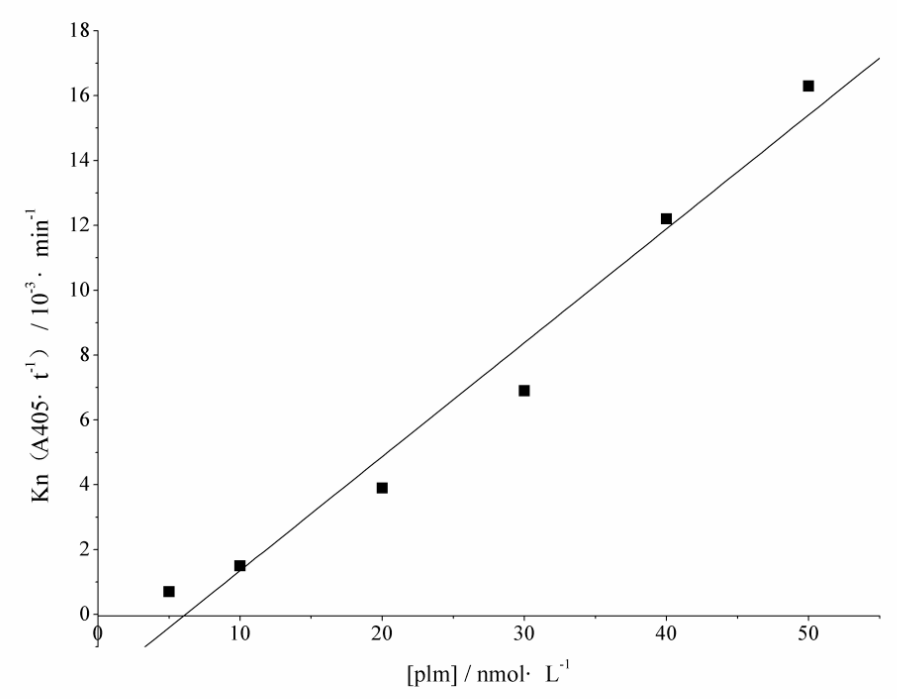

Figure 4. Plot of $K n$ versus [Plm].

\subsection{The Effect of FGFC1 on Km,plm of Pro-uPA Activation Plg}

$A_{405}$ and $t$ were the function of the second-order polynomial in the reaction of pro-uPA activated plasminogen, where $A$ is the coefficient of second-order polynomial $\left[A=0.5 \varepsilon \cdot L \cdot k_{\mathrm{cat}}, \mathrm{plm} \cdot v(\mathrm{plm})\right]$. The concentration of pro-uPA was constant in the reaction of pro-uPA/plasminogen/FGFC1. Figure 5 shows the coefficient of the quadratic term $\left(0.076 \times 10^{-4}-11.000 \times 10^{-4} t^{-2} / \mathrm{min}^{-2}\right)$ with different concentrations of FGFC1 $\left(0-40 \mu \mathrm{g} \cdot \mathrm{mL}^{-1}\right)$ and plasminogen $\left(30-110 \mathrm{nmol} \cdot \mathrm{L}^{-1}\right)$.

$v(\mathrm{plm})$ was obtained by the equation $(v(\mathrm{plm})=2 A / a)$ and double-reciprocal plot of initial velocity $v(\mathrm{plm})$ versus [plm], as shown in Figure 6. The slope of the line divided by intercept was

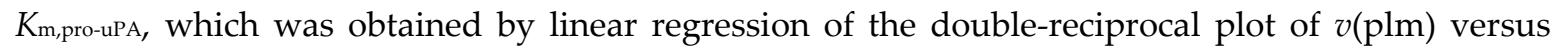

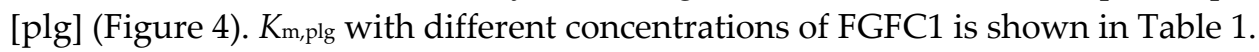

Table 1. The values of $K_{\mathrm{n}}$ at different concentration of plasmin.

\begin{tabular}{cc}
\hline $\mathbf{p l m} / \mathbf{n m o l}^{\cdot \mathbf{L}^{-\mathbf{1}}}$ & $\boldsymbol{K}_{\mathrm{n}}\left(A_{400} \cdot \mathbf{t}^{-\mathbf{1}}\right) / \mathbf{1 0}^{-\mathbf{3}} \cdot \mathbf{m i n}^{\mathbf{1}}$ \\
\hline 50 & 16.3 \\
40 & 12.2 \\
30 & 6.3 \\
20 & 3.9 \\
10 & 1.5 \\
5 & 0.7 \\
\hline
\end{tabular}




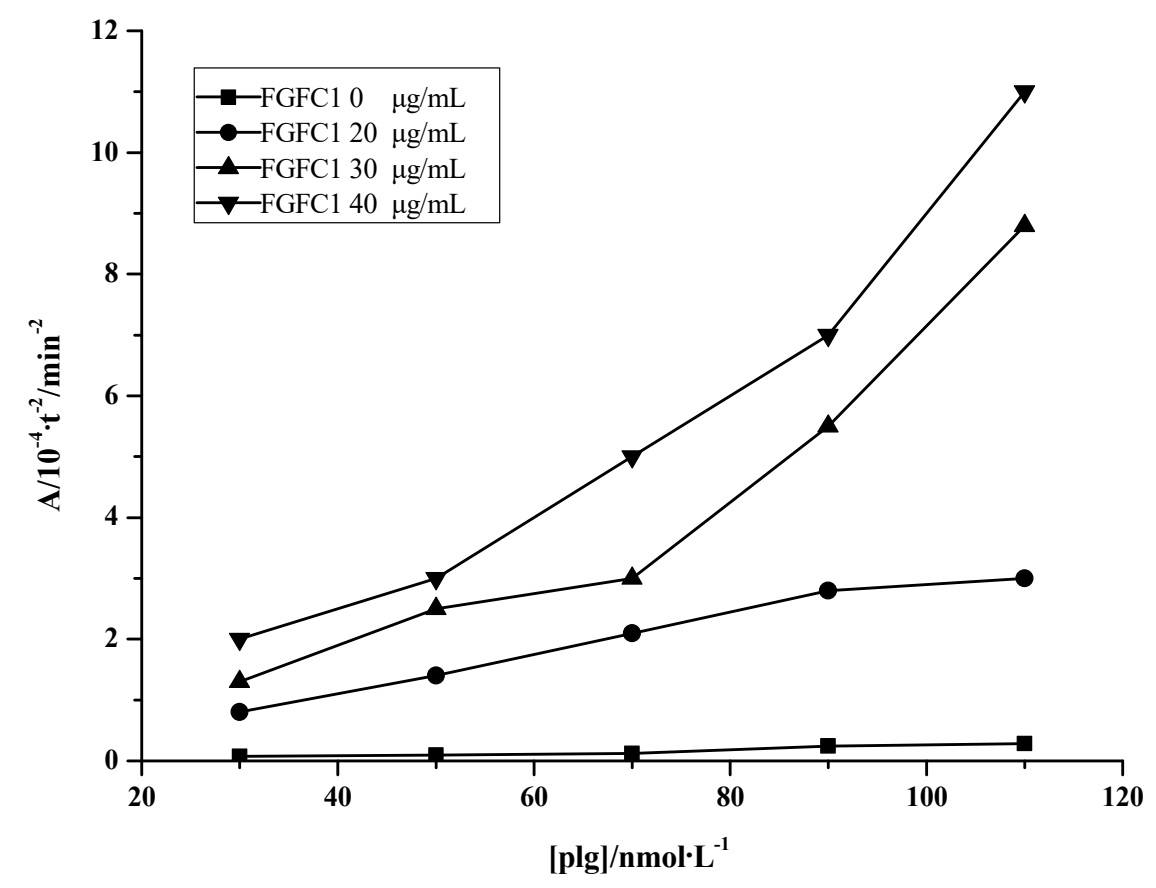

Figure 5. The coefficient of quadratic item value $A$ and $v(\mathrm{Plm})$ derived from $A$ at different concentrations of plasminogen.

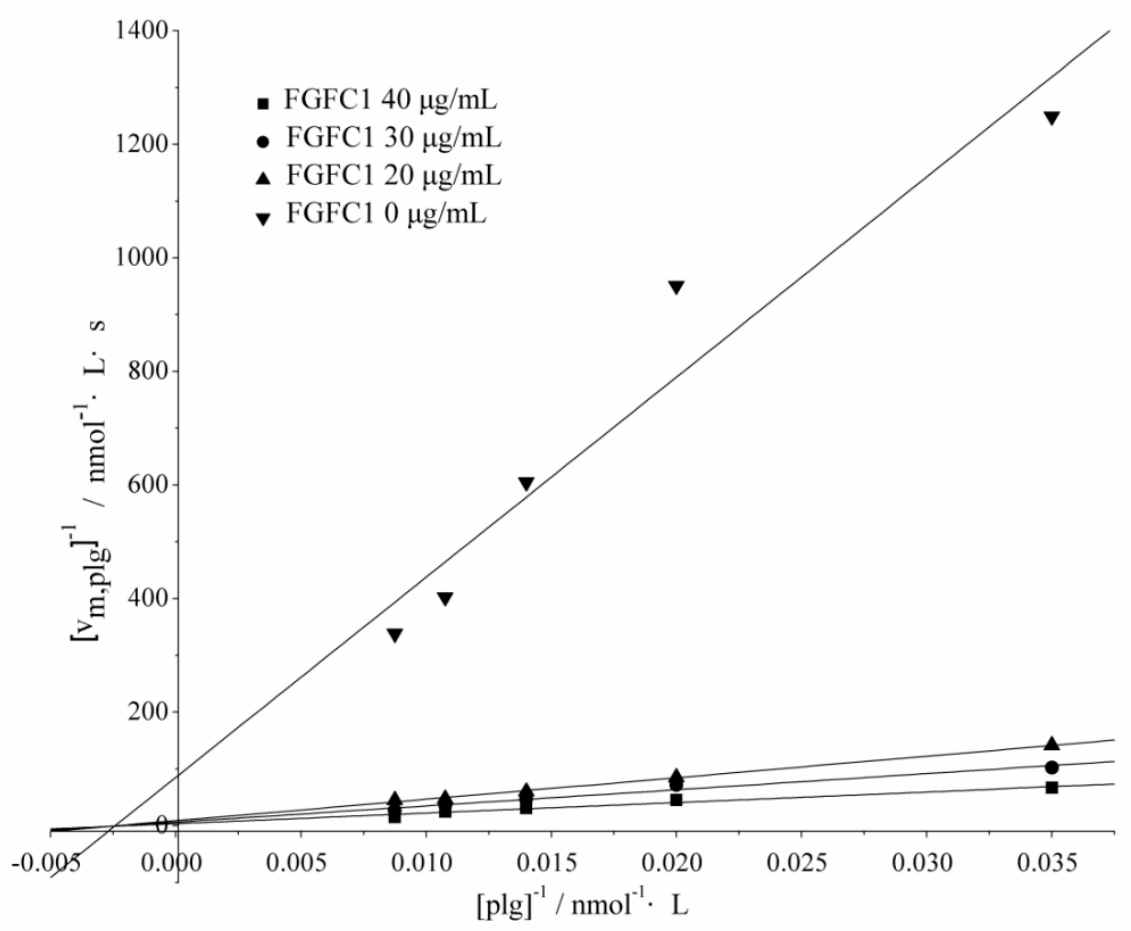

Figure 6. Double-reciprocal plot of initial velocity $v(\mathrm{plm})$ versus [plg].

\subsection{The Effect of FGFC1 on $k_{c a t, p r o-u P A}$ of Pro-uPA Activation Plg}

The curve of $A_{405-t}$ was polynomial regression in the reaction between pro-uPA and plasminogen, and the coefficient of the second-order item $A$ was obtained (Figure 7) as follows: $A=$ 
$0.5 \varepsilon \cdot L \cdot k_{\mathrm{cat}, \mathrm{plm}} \cdot v(\mathrm{plm})$. The concentration of plasminogen was constant in the reaction of pro-uPA/plasminogen/FGFC1. The coefficient of quadratic term $\left(0.050 \times 10^{-4}-5.000 \times 10^{-4} t^{-2} / \mathrm{min}^{-2}\right)$ with different concentrations of FGFC1 $\left(0-40 \mu \mathrm{g} \cdot \mathrm{mL}^{-1}\right)$ and pro-uPA $\left(10-40 \mathrm{nmol} \cdot \mathrm{L}^{-1}\right)$ is shown in Figure 7. $v(\mathrm{plm})$ was obtained according to the equation $[v(\mathrm{plm})=2 A / a]$ and the curve of $v \mathrm{~m}$ versus

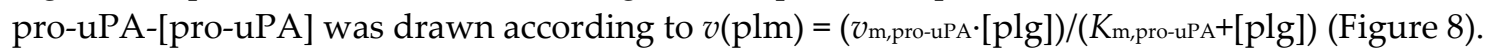

The slope of the line $k_{\text {cat,pro-uPA }}$ was yielded according to regressing linearly Figure 8 . When

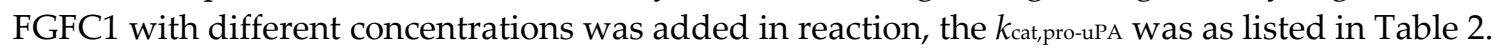

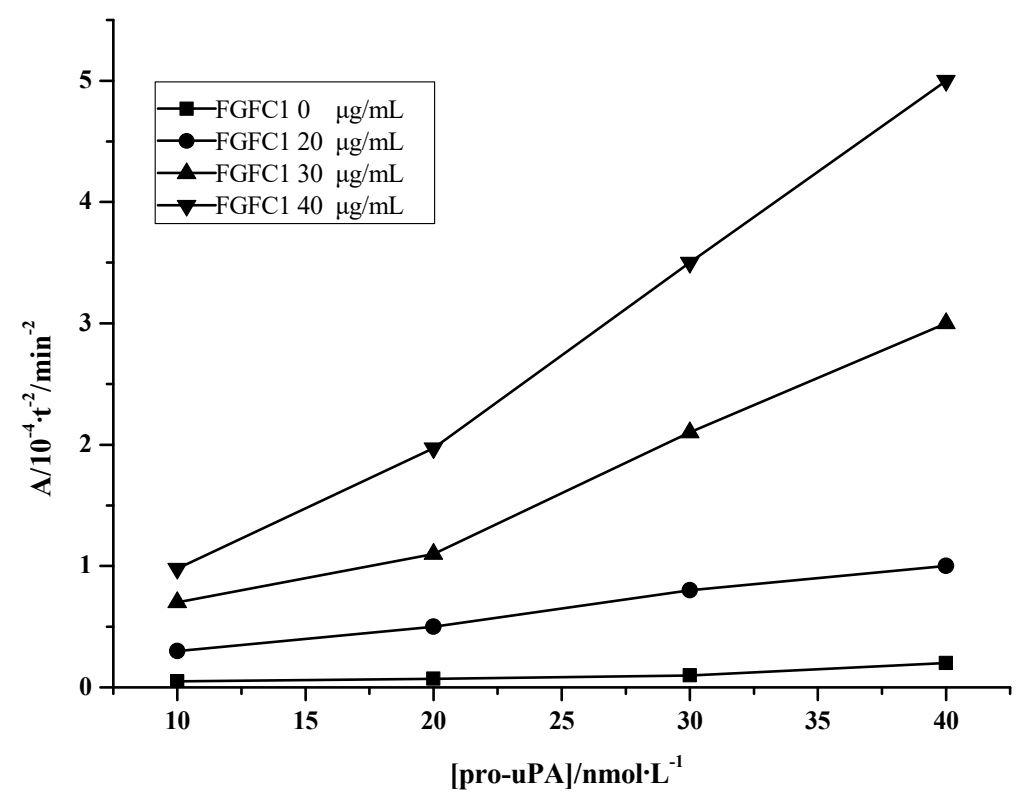

Figure 7. The coefficient of quadratic item value $A$ and $v(\mathrm{Plm})$ derived from $\mathrm{A}$ at different concentration of pro-uPA.

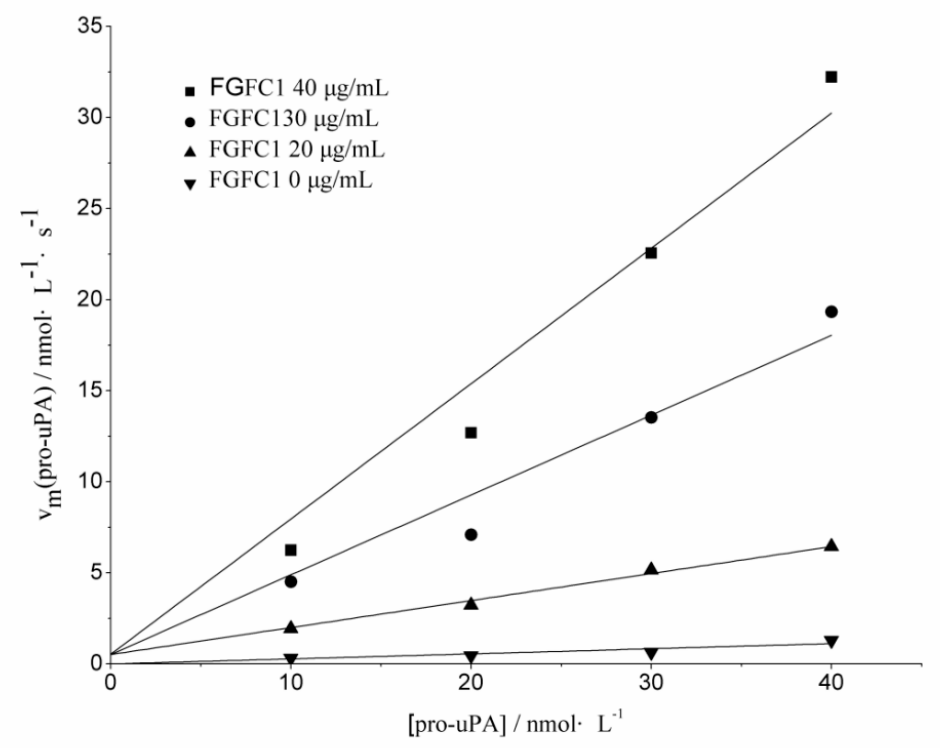

Figure 8. Plot of $v$ m,pro-uPA versus [pro-uPA]. 
Table 2. Kinetic constant (with different concentrations of FGFC1).

\begin{tabular}{cccc}
\hline FGFC1/ $\boldsymbol{\mu g} \cdot \mathbf{m L}^{-1}$ & \multicolumn{3}{c}{ Kinetic constant } \\
& $k_{\mathrm{cat}} / \mathrm{S}^{-1}$ & $K_{\mathrm{m}} / \mu \mathrm{mol} \cdot \mathrm{L}^{-1}$ & $k_{\mathrm{cat}} / k_{\mathrm{m}}$ \\
\hline 40 & 0.743 & 0.484 & 1.535 \\
30 & 0.438 & 0.439 & 0.998 \\
20 & 0.148 & 0.422 & 0.351 \\
0 & 0.028 & 0.413 & 0.067 \\
\hline
\end{tabular}

The plasmin hydrolyzed synthetic enzyme substrate S-2444 (pyro-Glu-Gly-Arg- $p \mathrm{NA} \cdot \mathrm{HCl}$ ) on the peptide bond of Arg-pNA and the steady-state rate of synthetic enzyme substrate degradation by plasmin were determined at various plasmin concentrations. The linear dependence between the plasmin concentrations and synthetic enzyme substrate degradation was obtained in a parameter $a$ $\left(a=\varepsilon \cdot L \cdot k_{\text {cat }, \text { plm }}=3.514 \times 10^{-4} A_{405} \cdot \mathrm{min}^{-1} \cdot \mathrm{nmol}^{-1}\right)$. The slope of the line was lower than a $=\varepsilon \cdot L \cdot k_{\text {cat,plm }}=3.4 \times$ $10^{5} A_{405} \cdot \mathrm{min}^{-1} \cdot \mathrm{mol}^{-1}$ where the slope was calculated by synthetic enzyme substrate S-2251 (H-D-Val-Leu-Lys-pNA·2HCl) and plasmin [14]. The enzymatic kinetic parameter was associated with both the amino acid sequence of the synthetic enzyme substrate and peptide bond. It was suggested that the 1000 folds by plasmin cleavage was via Lys-pane than Arg- $p$ NA.

The reaction of mutual activation between the single chain urokinase type plasminogen activator and plasminogen, the kinetic constant (single chain urokinase type plasminogen activator actives plasminogen) was as follows: $K_{\mathrm{m}}=0.413 \mu \mathrm{mol} \cdot \mathrm{L}^{-1}, k_{\mathrm{cat}}=0.028 \mathrm{~s}^{-1}$ and $K_{\mathrm{m}}=0.4-1.1 \mu \mathrm{mol} \cdot \mathrm{L}^{-1}, k_{\text {cat }}$ $=0.020-0.093 \mathrm{~s}^{-1}$ from a similar reaction system [5]. $K_{\mathrm{m}}$ and $k_{\text {cat }}$ were affected by plasminogen species of human, cat, dog, bovine, rabbit and horse. $K_{\mathrm{m}}$ and $k_{\text {cat }}$ of plasminogen was $0.69 \pm 0.07-1.40 \pm 0.26$ and $6.18 \pm 0.37-298.42 \pm 26.91$ by urokinase, respectively [15]. The range of $K_{m} / k_{\text {cat }}$ was from 5.8-80.7. The enzymatic kinetics parameters $K_{\mathrm{m}}$ and $k_{\text {cat }}$ of plasminogen were ascertained by the type of plasminogen activators, such as two chain urokinase type plasminogen activators, or tissue type plasminogen. The data of $K_{\mathrm{m}}, k_{\mathrm{cat}}$ and $K_{\mathrm{m}} / k_{\mathrm{cat}}$ of $0.413,0.028$ and 0.067 were reasonable values in the absence of FGFC1. It was shown that the intrinsic activity single chain urokinase type plasminogen activator had a torpid activity to cleavage plasminogen [16], which was an equitable physiological reaction.

In the reaction of pro-uPA activating plasminogen, $k_{\mathrm{cat}}$ and $k_{\mathrm{cat}} / K_{\mathrm{m}}$ increased significantly with increasing FGFC1 concentration. $k_{\text {cat }}$ and $k_{\text {cat }} / K_{\mathrm{m}}$ exhibited 26.5 -fold and 22.8 -fold increased activity at $40 \mu \mathrm{g} \cdot \mathrm{mL}^{-1}$ FGFC1 concentration, respectively. The results suggested that FGFC1 significantly improved the maximum catalytic efficiency and the total catalytic activity of pro-uPA. Moreover, $K_{\mathrm{m}}$ increased with increasing FGFC1 concentration, which indicated that FGFC1 slightly decreased the affinity activity of pro-uPA versus plasminogen.

The hydrophilicity of the reaction system changed after weakly acidic FGFC1 was added. Therefore, it was concluded that the $K_{\mathrm{m}}$ value increased slightly after the addition of FGFC1 was attributed to the change in solution micro-environment caused by FGFC1. The active center of scu-PA interacted with $\mathrm{Arg}^{560}-\mathrm{Val}^{561}$ near $\mathrm{K} 5$ of the plasminogen in the reaction of plasminogen and scu-PA. Scu-PA cleaved the Arg ${ }^{560}-V_{a l}{ }^{561}$ bond of plasminogen by intrinsic activity similar to uPA activity. FGFC1 accelerated the transformed efficiency that the single chain urokinase type plasminogen activator transformed the plasminogen. Moreover, fibrinolytic activity was enhanced by FGFC1, which changed the conformation of plasminogen $[17,18]$. It was concluded that FGFC1 changed the space structure near $\mathrm{K} 5$ and further formed the peptide fraction of $\mathrm{Arg}^{560} \mathrm{Val}^{561}$ of plasminogen, which consisted of the activated center of single chain urokinasetype plasminogen activator [13]. The urokinase from the single chain urokinase type plasminogen activator transforming plasminogen changed conform to plasmin easily in the reaction system. The results showed that the kinetic constants ( $k_{\text {cat }}$ and $k_{\mathrm{cat}} / \mathrm{K}_{\mathrm{m}}$ ) increased drastically in the reaction system.

$v_{\max }$ of the enzymatic reaction was given through expression to degradation of S-2444 (pyro-Glu-Gly-Arg-pNA·HCl) by urokinase associated with plasminogen and the single chain 
urokinase type plasminogen activator (Figure 9). $K_{\mathrm{m}}$ was a steady-state constant in absence of FGFC1 and presence of FGFC1 at the enzymatic reaction. At low values of S-2444 (pyro-Glu-Gly-Arg- $p \mathrm{NA} \cdot \mathrm{HCl}$ ), the initial velocity, $v_{i}$, rose almost linearly with increasing substrate. $K_{\mathrm{m}}$ was calculated in substrate concentration when $v_{\mathrm{i}}$ was one-half $v_{\text {max. }} k_{\mathrm{cat}}$ of the enzymatic reaction increased with the presence of FGFC1. FGFC1 had enhanced fibrinolysis via raising the value of $k_{\text {cat }} / K_{\mathrm{m}}$ at enzymatic kinetic performance.

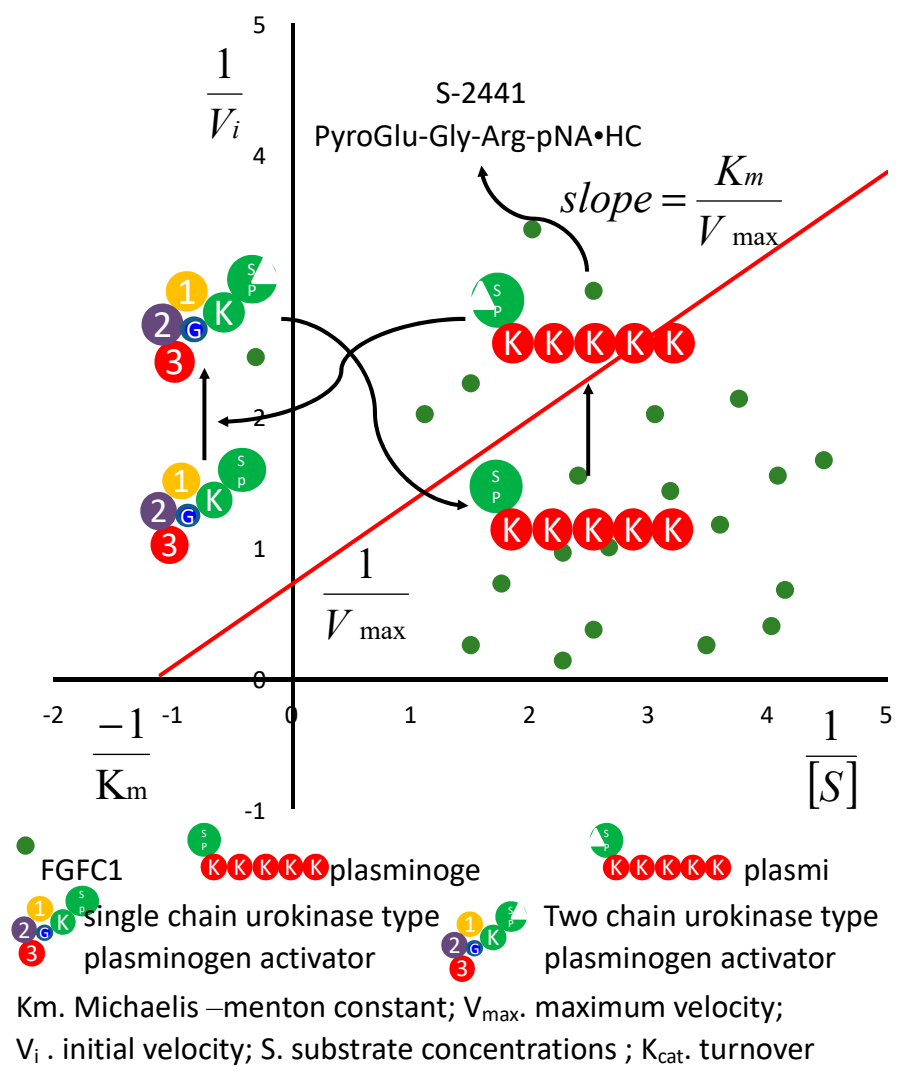

Figure 9. The diagrammatic sketch of FGFC1 enhancing fibrinolytic kinetics performance based on the reciprocal reaction of plasminogen and single chain urokinase type plasminogen activator.

\section{Materials and Methods}

\subsection{Materials}

Pro-uPA, plasminogen and plasmin were purchased from Sigma-Aldrich (China). The chromogenic s S-2444 and S-2251 were purchased from BioMed. Tris-HCl buffer (50 $\mathrm{mmol} \cdot \mathrm{L}^{-1}, 100$ mmol. $\mathrm{L}^{-1} \mathrm{NaCl}, \mathrm{pH}$ 7.4) and an enzyme-labeled instrument (SH-1000, CORONA) were used throughout the experiments. FGFC1 was isolated from the rare marine microorganism strain $S$. longispora FG216.

\subsection{Kinetic Theoretical Analysis of Plasminogen Activation [19]}

The reaction rate of pro-uPA was obtained by measuring the UV absorbance of $p$-NA at $405 \mathrm{~nm}$, which was produced by the plasmin-catalyzed hydrolytic reaction of the chromogenic substrate S-2444 [20].

The conversion of pro-uPA and plasminogen to plasmin and $p$-NA is represented by a sequence of two reactions which obey Michaelis-Menten kinetics, i.e.,

Reaction 1: 
pro-uPA+plg $\stackrel{K_{\mathrm{m}, \text { pro-uPA }}}{\longrightarrow}$ pro-uPA plg $\stackrel{k_{\text {cat, prouPA }}}{\longrightarrow}$ pro-uPA+plm

Reaction 2:

$\mathrm{plm}+\mathrm{S}-2444 \stackrel{K_{\mathrm{m}, \mathrm{plm}}}{\longleftrightarrow} \mathrm{plm} \cdot \mathrm{S}-2444 \stackrel{K_{\text {cat }, \mathrm{plm}}}{\longrightarrow} \mathrm{plm}+\mathrm{VLK}+p-\mathrm{NA}$

In Reaction 1, the rate of plasmin was generated by pro-uPA within the interval, which can be expressed as Equation (1). Therefore, at any time, $v_{t}(\mathrm{Plm})$ :

$$
v_{t}(P l m)=\frac{\mathrm{d}[\mathrm{Plm}] t}{\mathrm{~d} t}=\frac{V_{\mathrm{mPA}} \cdot[\mathrm{Plg}] t}{K_{\mathrm{mPA}}+[\mathrm{Plg}] t}
$$

As $v_{\mathrm{mPA}}=k_{\text {cat }}[\mathrm{PA}]$, the integrals toward $\mathrm{t}$ in Equation (1) are as follows:

$$
[\mathrm{Plm}]=\int \frac{\mathrm{d}[\mathrm{Plm}]}{\mathrm{d} t} \cdot \mathrm{dt}=\int \frac{k_{\mathrm{cat}, \mathrm{PA}} \cdot[\mathrm{PA}] \cdot[\mathrm{Plg}] t}{K_{\mathrm{mPA}}+[\mathrm{Plg}] t} \cdot \mathrm{dt}
$$

In Equation (2), $[\mathrm{Plg}]_{t}=[\mathrm{Plg}]_{0}-\int_{0}^{\mathrm{t}} v \mathrm{t}(\mathrm{P} l \mathrm{~m}) \cdot \mathrm{d} t<[\mathrm{Plg}]_{0}-v_{0}(\mathrm{Plm}) \cdot t$. According to the references, $K_{\mathrm{m}}$ and $k_{\text {cat }}$ of plasminogen activation by natural scu-PA were located in the range of range in 0.4-1.1 $\mu \mathrm{mol} \cdot \mathrm{L}^{-1}$ and $0.02-0.093 \mathrm{~s}^{-1}$, respectively [21-23]. Under the condition of $[\mathrm{Plg}]_{0}=0.1 \mu \mathrm{mol} \cdot \mathrm{L}^{-1},[\mathrm{PA}]=$ $1.4 \times 10^{-9} \mathrm{~mol} \cdot \mathrm{L}^{-1}, v_{0}(\mathrm{Plm})$ was calculated $\left(0.02 \times 1.4 \times 10^{-9} \times 0.1 \times 10^{-6} /\left[(0.4+0.1) \times 10^{-6}\right]=5.6 \times 10^{-12}\right.$ $\left.\mathrm{mol} \cdot \mathrm{L}^{-1} \cdot \mathrm{s}^{-1}\right) \cdot 0.1 \mu \mathrm{mol} \cdot \mathrm{L}^{-1}$ plasmin, which was approximately consumed about $17,857 \mathrm{~s}$ or $297 \mathrm{~min}$, was ignored in comparison with produced plasmin in $2400 \mathrm{~s}$. Therefore, $[\mathrm{Plg}]_{t}=[\mathrm{Plg}]_{0}=[\mathrm{Plg}]$ in $2400 \mathrm{~s}$ was substituted into Equation (2), resulting in the following:

$$
[\mathrm{Plm}]=\int \frac{k_{\mathrm{cat}, \mathrm{PA}} \cdot[\mathrm{PA}] \cdot[\mathrm{Plg}]}{K_{\mathrm{mPA}}+[\mathrm{Plg}]} \cdot \mathrm{d} t=\frac{k_{\mathrm{cat}, \mathrm{PA}} \cdot[\mathrm{PA}] \cdot[\mathrm{Plg}]}{K_{\mathrm{mPA}}+[\mathrm{Plg}]} \cdot t+C_{1}
$$

As $\frac{k_{\text {cat }}, \mathrm{PA} \cdot[\mathrm{PA}] \cdot[\mathrm{Plg}]}{K_{\mathrm{m}} \mathrm{PA}+[\mathrm{Plg}]}=v(\mathrm{Plm})$ and $C_{1}$ is essentially constant, Equation (3) can be described as follows:

$$
[\mathrm{Plm}]=v(\mathrm{Plm}) \cdot t \cdot \mathrm{C}_{1}
$$

From Reaction 2, it can be seen that generation rate of $p$-NA can be described by the following rate:

$$
\mathrm{v}(p-\mathrm{NA})=\frac{v_{\mathrm{mPLm}} \cdot[\mathrm{S}-2251]}{K_{\mathrm{mPLm}} \cdot[\mathrm{S}-2251]}=\frac{k_{\mathrm{cat}}[\mathrm{PLm}] \cdot[\mathrm{S}-2251]}{K_{\mathrm{mPLm}}+[\mathrm{S}-2251]}
$$

Under the experimental conditions, $K_{\mathrm{m}, \mathrm{Plm}}$ of plasmin hydrolysis S-2444 $\left(250 \mu \mathrm{mol} \cdot \mathrm{L}^{-1}\right)$ was far less than S-2444 $\left(1.5 \mathrm{mmol} \cdot \mathrm{L}^{-1}\right)$. Therefore, $K_{\mathrm{m}, \mathrm{Plm}}$ was deleted and Equation (5) reduced to $v(\mathrm{p}-\mathrm{NA})=$ $K_{\text {cat,PIm }} \cdot[\mathrm{Plm}]$, which integration yielded:

$$
[p-\mathrm{NA}]=\int \frac{\mathrm{d}[\mathrm{p}-\mathrm{NA}]}{\mathrm{d} t} \cdot d t=\int k_{\mathrm{cat}, P L m} \cdot[P L m] \cdot \mathrm{d} t
$$

When Reaction 1 and 2 happened at the same time, [Plm] is the function of $t$, substitution of Equation (4) in Equation (6) gave:

$$
\left.\left.[p-\mathrm{NA}]=\int k_{\mathrm{cat}, \mathrm{Plm}} \cdot[\mathrm{v}(\mathrm{Plm})]\right] \cdot \mathrm{t}+\mathrm{C} 1\right] \cdot \mathrm{d} t=\frac{1}{2} k_{\mathrm{cat}, \mathrm{Plm}} \cdot v(\mathrm{Plm}) \cdot \mathrm{t}^{2}+k_{\mathrm{cat}, \mathrm{Plm}} \cdot \mathrm{C} 1 \cdot \mathrm{t}+\mathrm{C} 2
$$

The most absorbance of chromophore group $p$-NA is $A_{405}$, which was expressed as: $\varepsilon \cdot L \cdot[p$-NA] where $L$ is the height of the reaction liquid in 96-hole plate; and $\varepsilon$ is molar extinction coefficient of $p$-NA. Therefore, substitution of Equation (7) in $A_{405}=\varepsilon \cdot L \cdot[p-\mathrm{NA}]$ gave:

$$
A_{405}=0.5 \cdot \varepsilon \cdot L \cdot k_{\mathrm{cat}, \mathrm{PIm}} \cdot \mathcal{V}_{\{\mathrm{PIm}\}} \cdot t^{2}+\varepsilon \cdot L \cdot k_{\mathrm{cat}, \mathrm{PIm}} \cdot C_{1} \cdot t+\varepsilon \cdot L \cdot C_{2}
$$


Equation (8) can be simplified as follows:

$$
A_{405}=A \cdot t^{2}+B \cdot t+C
$$

where $C=\varepsilon \cdot L \cdot C_{2} ; B=\varepsilon \cdot L \cdot k_{\mathrm{cat}, \mathrm{PIm}} \cdot C_{1} ; A=0.5 \cdot \varepsilon \cdot L \cdot k_{\mathrm{cat}, \mathrm{Plm}} \cdot \nu_{\{\mathrm{Plm}\}}$,

From Equation (9), it can be seen that the relationship between $A_{405}$ and $t$ meets up with second-order polynomial when Reactions 1 and 2 occurred simultaneously.

$A$ is the coefficient of second-order polynomial. The standard curve of S-2444 of plasmin hydrolysis is introduced to delete $\varepsilon \cdot L \cdot k_{\mathrm{cat}, P I m}$ as follows:

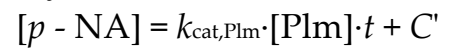

where $[\mathrm{Plm}]$ is constant. Consequently, $A_{405}$ was expressed as:

$$
A_{405}=\varepsilon \cdot L \cdot k_{\mathrm{cat}, \mathrm{PLm}} \cdot[\mathrm{Plm}] \cdot t+C_{3}
$$

Equation (10) can be simplified as follows:

$$
A_{405}=K \cdot t+C_{3}
$$

where $C_{3}=\varepsilon \cdot L \cdot C^{\prime}, K=\varepsilon \cdot L \cdot k_{\mathrm{cat}, \mathrm{PLm}} \cdot[\mathrm{Plm}]$

From Equation (10), it can be concluded that the scatter diagram of S-2444 by plasmin hydrolysis is made with constant concentration of $[\mathrm{Plm}]_{\mathrm{n}}$, and the slope on $[\mathrm{Plm}]_{\mathrm{n}}$ is obtained as follows:

$K_{\mathrm{n}}=\varepsilon \cdot L \cdot k_{\mathrm{cat}, P \mathrm{Plm}} \cdot[\mathrm{Plm}]_{\mathrm{n}}$

The second scatter diagram was drawn and the equation of $K_{\mathrm{n}}=\varepsilon \cdot L \cdot \mathrm{kat}_{\mathrm{calm}} \mathrm{Pl} \cdot[\mathrm{Plm}]_{\mathrm{n}}$ obtained. If the slope is $a$, it can be concluded that $a=\varepsilon \cdot L \cdot k_{\mathrm{cat}, \mathrm{PIm}}$. Moreover, $A=0.5 \cdot \varepsilon \cdot L \cdot k_{\mathrm{cat}, \mathrm{PIm}} \cdot \boldsymbol{v}_{\{\mathrm{PIm}\}}$ was substituted in $K_{\mathrm{n}}=\varepsilon \cdot L \cdot k_{\mathrm{cat}, P \mathrm{Pm}} \cdot[\mathrm{Plm}] \mathrm{n}$.

$$
v\{\mathrm{Plm}\}=2 \mathrm{~A} / \mathrm{a}
$$

According to Equation (12), $v_{(\mathrm{Plm})}$ was calculated with different concentrations. Then, according to the method of Line weaver-Burk, the equation can be obtained as follows:

$$
\frac{1}{v(\mathrm{Plm})}=\frac{K_{\mathrm{mPA}}}{v_{\mathrm{mPA}}} \cdot \frac{1}{[\mathrm{Plg}]}+\frac{1}{v_{\mathrm{mPA}}}
$$

At the same time, the corresponding linear regression line was also obtained. The slope of $a$ straight line divided by intercept was the value of $K_{\mathrm{m}, \mathrm{PA}} \cdot \mathrm{v}_{\mathrm{m}, \mathrm{PA}}$, which can be calculated with a fixed concentration of $[\mathrm{PA}]_{n}$ according to the equation as follows:

$$
v(\mathrm{Plm})=\frac{v_{\mathrm{mPA}} \cdot[\mathrm{Plg}]}{K_{\mathrm{mPA}}+[\mathrm{Plg}]}
$$

Then the curve is drawn by $v_{\mathrm{m}, \mathrm{PA}}$ versus [PA] and the equation is obtained as follows:

$$
v_{\mathrm{m}, \mathrm{PA}}=k_{\mathrm{cat}, \mathrm{PA}} \cdot[\mathrm{PA}]
$$

The corresponding line is also obtained, and the slope is $k_{\text {cat,PA. }}$

\subsection{Determination of Standard Curve by S-2444 Hydrolysis}

The reaction mixture contained chromospheres substrates S-2444, Tris- $\mathrm{HCl}$ buffer $(50 \mathrm{mmol} / \mathrm{L}$, $\left.100 \mathrm{mmol} / \mathrm{L} \mathrm{NaCl}, \mathrm{pH} 7.4,25{ }^{\circ} \mathrm{C}\right)$, and plasmin $\left(5,10,20,30,40\right.$, and $\left.50 \mathrm{nmol} \cdot \mathrm{L}^{-1}\right)$ was incubated in a 96-well plate with a round bottom at $37{ }^{\circ} \mathrm{C}$ for $90 \mathrm{~min}$. The absorbance of reaction mixture was determined continuously at $37^{\circ} \mathrm{C}$ for $44 \mathrm{~min}$. From the equation $K_{n}=\varepsilon \cdot L \cdot k_{\mathrm{cat}, \mathrm{Plm}} \cdot[\mathrm{Plm}]_{n}, K_{n}$ (the slope of each curve) was obtained by the linear regression of $A_{405 n m-t}$ (Figure 3).

\subsection{Determination of $K_{m, p r o-u P A}$ of Plasminogen Activation by Pro-uPA}

Pro-uPA (10 nmol. $\left.\mathrm{L}^{-1}\right)$ was incubated with a range of concentrations $(30,50,70,90$, and 110 $\left.\mathrm{nmol} \cdot \mathrm{L}^{-1}\right)$ of plasminogen in $0.8 \mathrm{mmol} \cdot \mathrm{L}^{-1} \mathrm{~S}-2444$, Tris- $\mathrm{HCl}(50 \mathrm{mmol} / \mathrm{L}, 100 \mathrm{mmol} / \mathrm{L} \mathrm{NaCl}, \mathrm{pH} 7.4,25$ $\left.{ }^{\circ} \mathrm{C}\right)$, and FGFC1 $(20,30$, and $40 \mu \mathrm{g} / \mathrm{mL})$ at $37^{\circ} \mathrm{C}$ for $90 \mathrm{~min}$. The absorbance of the reaction mixture 
was determined continuously at $37^{\circ} \mathrm{C}$ for $44 \mathrm{~min}$. The concentration of pro-uPA was constant in the reaction of pro-uPA/plasminogen/FGFC1. The Michaelis constant $\left(K_{\mathrm{m}, \text { pro-uPA }}\right)$ was determined from Line weaver-Burk plots with a computerized program (Figure 5).

\subsection{Determination of $k_{c a t, p r o-u P A}$ of Plasminogen Activation by Pro-uPA}

The reaction mixture was made up of pro-uPA $\left(20,30\right.$, and $\left.40 \mathrm{nmol} \cdot \mathrm{L}^{-1}\right), 0.8 \mathrm{mmol} \cdot \mathrm{L}^{-1} \mathrm{~S}-2444$, Tris- $\mathrm{HCl}$ buffer $\left(50 \mathrm{mmol} / \mathrm{L}, 100 \mathrm{mmol} / \mathrm{L} \mathrm{NaCl}, \mathrm{pH} 7.4,25^{\circ} \mathrm{C}\right)$, and FGFC1 $(10,20,30$, and $40 \mu \mathrm{g} / \mathrm{mL})$ at $37^{\circ} \mathrm{C}$. The absorbance of the reaction mixture was determined continuously at $37^{\circ} \mathrm{C}$ for $44 \mathrm{~min}$. The concentration of plasminogen was constant in the reaction of pro-uPA/plasminogen/FGFC1. The Michaelis constant ( $\left.K_{\text {cat,pro-upA }}\right)$ was determined from Line weaver-Burk plots with a computerized program (Figure 7).

\section{Conclusions}

In this paper, the enzymatic kinetics of fibrinolysis was described based on an enzymatic reaction of a chromogenic-substrate associated with $p$-nitroaniline $(p-\mathrm{NA}) . k_{\mathrm{cat}}$ and $k_{\mathrm{cat}} / K_{\mathrm{m}}$ increased significantly with an increase in FGFC1 concentration. Moreover, $k_{\text {cat }}$ and $k_{\text {cat }} / k_{\mathrm{m}}$ exhibited 26.5 -fold and 22.8-fold enhanced activity at a concentration of $40 \mu \mathrm{g} \cdot \mathrm{mL}^{-1}$ of FGFC1, respectively. The results suggested that FGFC1 significantly improved the maximum catalytic efficiency and the total catalytic activity of fibrinolysis base on the reciprocal activation of pro-uPA and plasminogen. $K_{\mathrm{m}}$ increased with increasing FGFC1 concentration, which indicated that FGFC1 slightly decreased the affinity activity of the pro-uPA and plasminogen versus enzyme substrate. The marine bisindole alkaloid FGFC1 enhanced fibrinolysis, which was taken on enzymatic kinetic characteristics. It is possible that FGFC1 is a potential thrombolytic agent in the future.

Acknowledgments: The work was supported by the National Natural Science Foundation of China (No. 81502955), the Doctoral Scientific Research Foundation of Shanghai Ocean University (No. A2030214300077), the Young Teachers Training Program of Shanghai (No. A12056160002), the Plan of Innovation Action in Shanghai (No. 14431906000), and the Project Funded by Jiangsu Key Laboratory of Marine Pharmaceutical Compound Screening.

Author Contributions: R.H. Guo and D. Duan performed data analysis and manuscript preparation; S.T Hong performed experiment data collection; Y. Zhou and F. Wang was responsible for drawing; S.J. Wang tested structure of FGFC1; W.H. Wu and B. Bao were the project leader, organizing and guiding the experiments and manuscript writing.

Conflicts of Interest: The authors declare no conflict of interest.

\section{References}

1. Ellis, V.; Scully, M.F.; Kakkar, V.V. Plasminogen activation initiated by single-chain urokinase-type plasminogen activator. Potentiation by U937 monocytes. J. Biol. Chem. 1989, 264, 2185-2188.

2. Schuster, V.; Hügle, B.; Tefs, K. Plasminogen deficiency. J. Thromb. Haemost. 2008, 5, 2315-2322.

3. Zhang, Y.; Wisner, A.; Xiong, Y.; Bon, C. A novel plasminogen activator from snake venom. Purification, characterization, and molecular cloning. J. Biol. Chem. 1995, 270, 10246-10255.

4. Mirshahi, M.; Soria, J.;Lijnen, H.R.;Fleury, V.; Bertrand, O.; Drouet, L.; Caen, J.P.; Soria, C. A monoclonal antibody directed against an epitope in the $\mathrm{NH}_{2}$-terminal region of native human plasminogen induces a modification of its functional properties. Fibrinolysis Proteol. 1997, 11, 155-163.

5. Lijnen, H.R.; Hoef, B.V.; De, C.F.;Collen, D. Effect of fibrin-like stimulators on the activation of plasminogen by tissue-type plasminogen activator (t-PA)--studies with active site mutagenized plasminogen and plasmin resistant t-PA. Thromb. Haemost. 1990, 64, 61-68.

6. Goretzki, L.; Schmitt, M.; Mann, K.; Calvete, J.; Chucholowski, N.; Kramer, M.; Guenzler, W.A.; Jaenicke, F.; Graeff, H. Effective activation of the proenzyme form of the urokinase-type plasminogen activator (pro-uPA) by the cysteine protease cathepsin L. FEBS Lett. 1992, 297, 112-118.

7. Marten, F.; Clifford, T.; Mary, B.D.; Shan, W.; Ruth, L.; David, A.D. Increased expression of urokinase during atherosclerotic lesion development causes arterial constriction and lumen loss, and accelerates lesion growth. Proc. Natl. Acad. Sci. 2002, 99, 10665-10670. 
8. Youn, U.J.; Lee, J.Y.; Kil, Y-S.; Han, A-R.; Cha, E-K.; Ryu, S.Y.; Seo, K.; Identification of new pyrrole alkaloids from the fruits of Lyciumchinense. Arch. Pharm. Res. 2016, 39, 321-327.

9. Tian, L.; Teng, X.; Zhong, C.; Xie, Y. Chemical constituents from the barks of Swietenia macrophylla. Gen. Chem. 2015, 1, 22-25.

10. Su, T.; Wu, W.; Yan, T.; Zhang, C.; Zhu, Q.; Bao, B. Pharmacokinetics and tissue distribution of a novel marine fibrinolytic compound in Wistar rat following intravenous administrations. J. Chromatogra. B 2013, 942, 77-82.

11. Yan, T.; Wu, W.H.; Su, T.W.; Chen, J.J.; Zhu, Q.G.; Zhang, C.Y.; Wang, X.Y.; Bao, B. Effects of a novel marine natural product: pyranoindolone alkaloid fibrinolytic compound on thrombolysis and hemorrhagic activities in vitro and in vivo. Arch. Pharm. Res. 2015, 38, 1530-1540.

12. Wang, G.; Wu, W.H.; Zhu, Q.G.; Fu, S.Q.; Wang, X.Y.; Hong, S.T.; Guo, R.H.; Bao, B. Identification and fibrinolytic activity of an indolone derivative isolated from a rare marine fungus Stachybotrys longispora FG216. Chin. J. Chem. 2015, 33, 1089-1095.

13. Hong, S.T.; Wu, W.H.; Zhou, Y.;Yan, T.; Zhou, Y.; Bao, B. Effect of indolone compound from marine microbial sources on conformation characteristics of fibrinolytic activity factor. Chin. J. Mar.Drugs 2015, 31,59-66.

14. Dang, X.; Ji, J.G.; Ru, Q.; Zhang, J.X.; Yu, M.M.; Ru, B.G. A new method of kinetic study on the reaction of plasminogen activation. Chin. J. Biochem. Mol. Biol. 2001, 17, 75-79.

15. Wohl, R.C.; Sinio, L.; Summaria, L.; Robbins, K.C. Comparative activation kinetics of mammalian plasminogens. Biochim. Et Biophys. Acta 1983, 745, 20-31.

16. Koudelka, S.; Mikulik, R.; Mašek, J.; Raška, M.; Knotigová, P.T.; Turánek, M.J. Liposomal nanocarriers for plasminogen activators. J. Controlled Release 2016, 227, 45-57.

17. Lee, C.H.; Park, K.J.; Kim, S.J.; Kwon, O.; Jeong, K.J.; Kim, A.; Kim, Y.S. Generation of bivalent and bispecifickringle single domains by loop grafting as potent agonists against death receptors 4 and 5 . J. Mol. Biol. 2011, 411, 201-219.

18. Behrens, M.A.; Botkjaer, K.A.; Goswami, S.; Oliveira, C.L.P.; Jensen, J.K.; Schar, C.R.; Declerck, P.J.; Peterson, C.B.; Andreasen, P.A.; Pedersen, J.S. Activation of the zymogen to urokinase-type plasminogen activator is associated with increased interdomain flexibility original research article. J. Mol. Biol. 2011, $411,417-429$.

19. Fares, A.E.; David, C.; Marie, R. Kinetic analysis of plasminogen activator inhibitor type-2: urokinase complex formation and subsequent internalisation by carcinoma cell lines. Exp. Cell Res. 2004, 297, 259-271.

20. Stump, D.C.; Lijnen, H.R.; Collen, D. Purification and characterization of single-chain urokinase-type plasminogen activator from human cell cultures. J. Biol. Chem. 1986, 261, 1274-1278.

21. Collen, D.; Zamarron, C.; Lijnen, H.R.; Hoylaerts, M. Activation of plasminogen by prourokinase. II. Kinetics. J. Biol. Chem. 1986, 261, 1259-1266.

22. Lijnen, H.R.; Zamarron, C.; Blaber, M.; Wrinkler, M.E.; Ollen, D. Activation of plasminogen by pro-urokinase.I. Mechanism. J. Biol. Chem. 1986, 261, 1253-1258.

23. Liu, J.N.; Gurewich, V. The kinetics of plasminogen activation by thrombin-cleaved prourokinase and promotion of its activity by fibrin fragment E-2 and by tissue plasminogen activator. Blood, 1993, 81, 980-987. 